\title{
Atención a la diversidad sexual
}

\section{Criterios educativos}

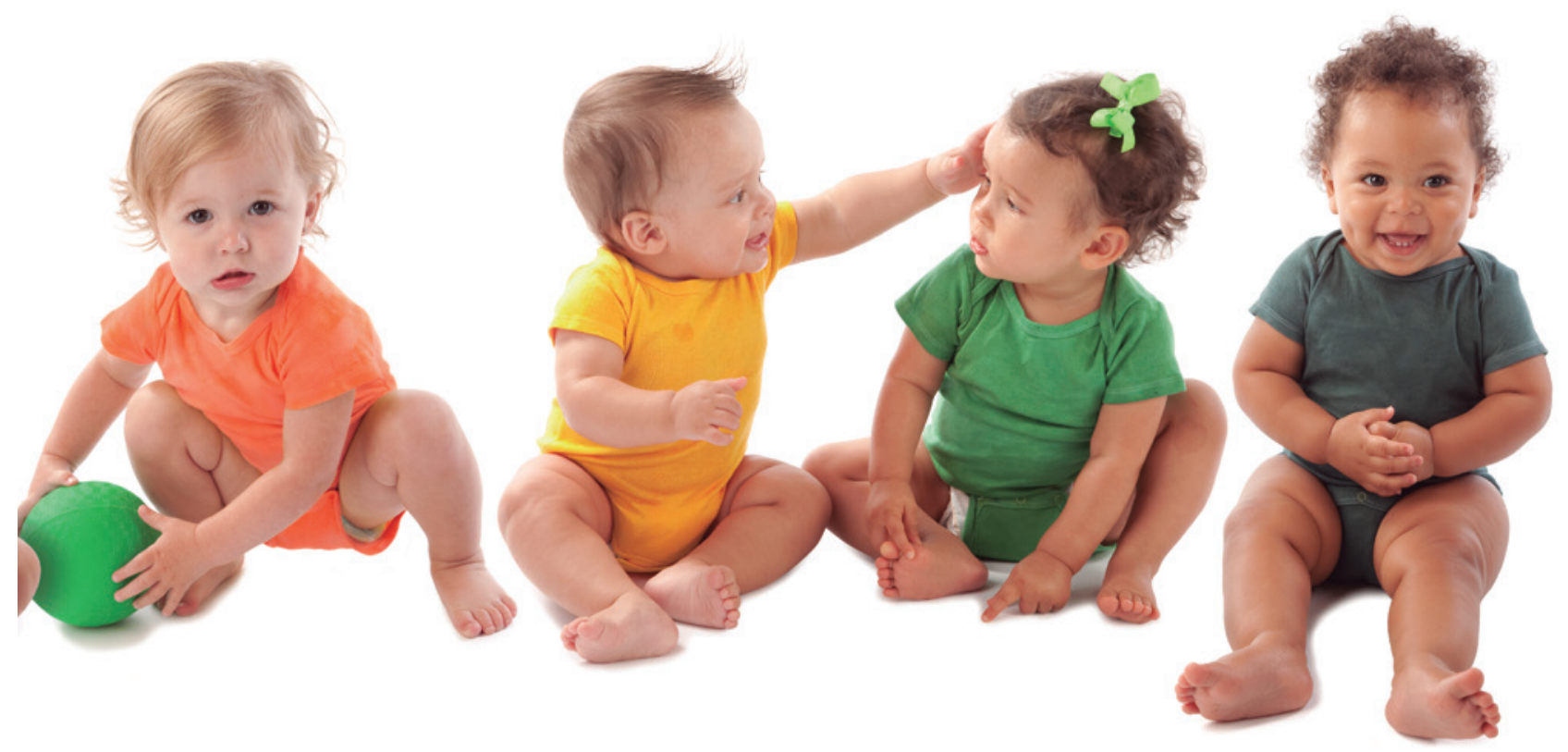

La atención a la diversidad sexual en los

centros de enseñanza requiere no sólo unas actuaciones concretas, sino unos criterios educativos claros que integren las actuaciones a nivel individual, de aula y de centro, con el

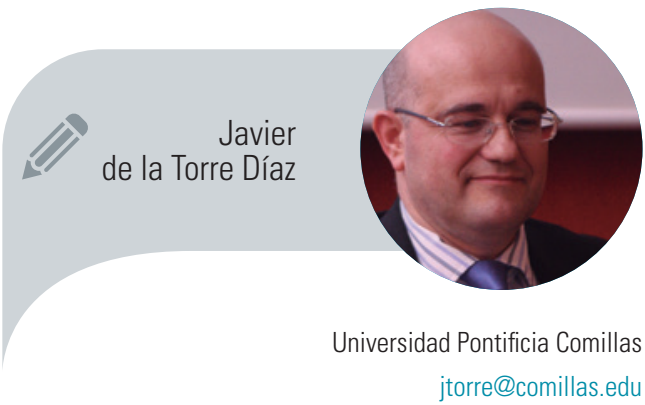
alumnado, con el profesorado y las familias. Expondremos estos criterios educativos en cuatro niveles. 


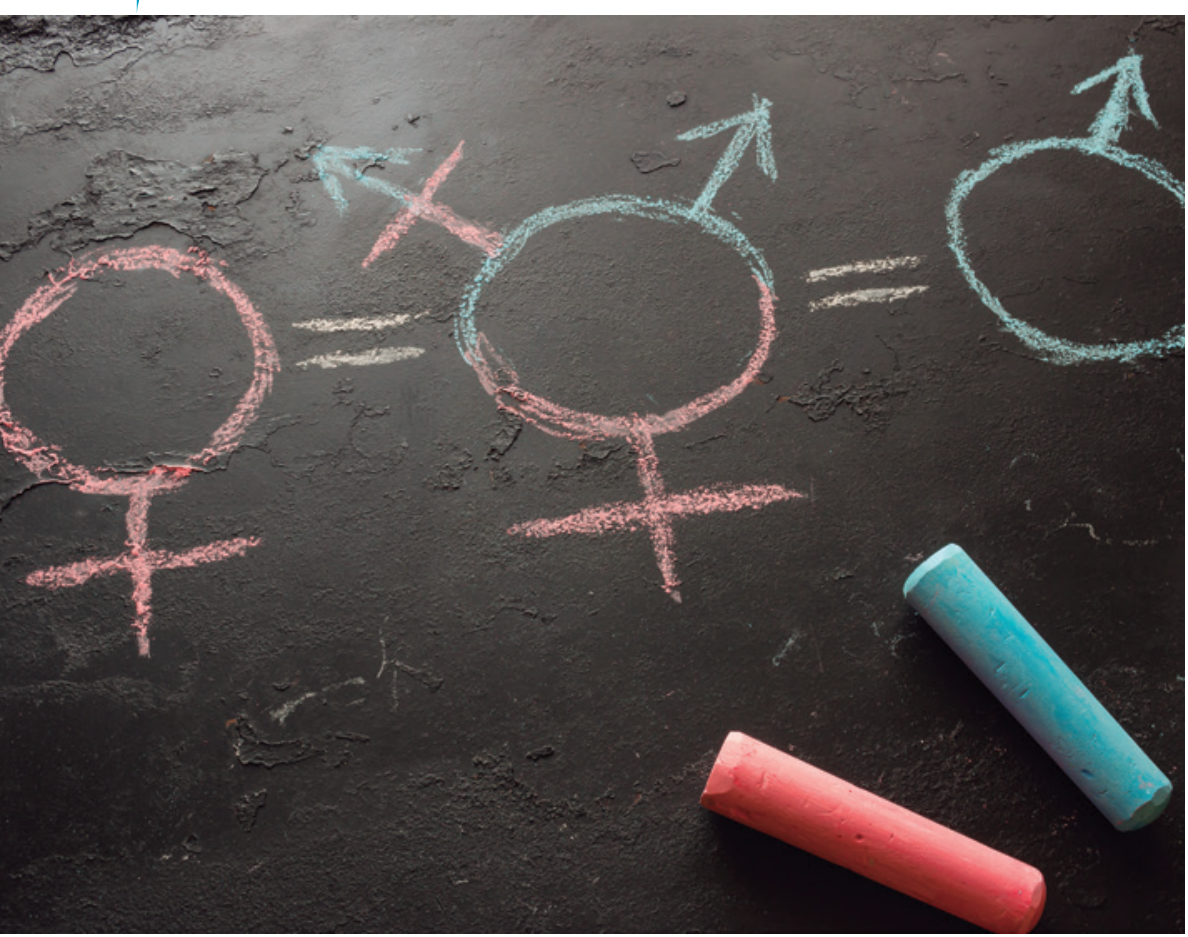

\section{Criterios básicos}

Educarse antes que educar. Lo primero es reconocer las propias dificultades ante la diversidad sexual. Hay que atreverse a concienciar la propia historia y las propias creencias. Tenemos que reconocer que muchos no tenemos en este terreno los conflictos resueltos. Sólo si reconozco mi actitud no muy positiva hacia la diversidad sexual puedo formarme para entrar en estos terrenos. Todos estamos en proceso y todos tenemos que educarnos para educar. La educación en la diversidad es una tarea de todos los profesores y de todo el personal del centro (monitores de actividades extraescolares, personal de administración, etc.). La diversidad es un tema transversal y no sólo de aulas. No es sólo una tarea de los "especialistas" y de los profesores y alumnos homosexuales y transexuales.

Conocimiento frente a ignorancia. Lo primero es proporcionar información seria, rigurosa, científica, actualizada. Todos tienen derecho a la información. Hay que integrar la diversidad sexual en la historia, el arte, la literatura, la ciencia, la biología, las ciencias de la religión, la ética. No se pueden silenciar en estas disciplinas algunos datos claros. El primer paso es reconocer y normalizar la diversidad. Hay que reconocer la diversidad en el mundo actual. Entre un 4 y un $10 \%$ de la población española es homosexual y más de $1 \%$ de la población está afectada por estados intersexuales. Hacer visible la diversidad sexual en la sociedad es importante. El primer reto educativo es acercar la realidad social y evitar la invisibilidad. No hay que presuponer la heterosexualidad de todos los profesores y alumnos, no hay que hablar como si fueran todos heterosexuales. Existe en los adolescentes, en los profesores, en las familias. Hoy es importante no ocultar la realidad. El silencio es el que lleva a los chantajes, dobleces, distorsiones y al sufrimiento de muchos. Hay silencios que se viven por parte de las propias personas, de sus compañeros, de sus profesores o incluso de las familias que son demoledores y que acarrean mucho sufrimiento. Hay que cuidar que en los libros de texto, materiales educativos y ejemplos en clase no se invisibilicen ciertas situaciones familiares y de diversidad sexual. Hay que ayudar a vencer este temor a la diversidad y capacitar emocionalmente a las personas que se bloquean. La diversidad altera a muchas personas que no tienen del todo su sexualidad bien integrada. Lo mejor siempre es asumir la realidad, lo cual implica combatir la falsa información y los prejuicios. Hay muchas maneras de ser gay y lesbiana, de vivir la disforia de género.

Comunicación frente a silencio. La escuela tiene el deber de crear espacios adecuados para que se puedan comunicar los sentimientos más íntimos. Tratar los temas con normalidad hace que no se vivan como un tabú o algo extraño. Es fundamental la creación de espacios claros de escucha y atención de las búsquedas, inquietudes, problemas, dudas de alumnos y profesores en este tema. Cuando se haya consolidado la identidad es necesario crear espacios para no sentirse en minoría, poderse expresar corporalmente en algún aspecto, espacios donde su afectividad sea aceptada y no explotada, espacios donde ser reconocido con naturalidad y sin temor y espacios donde la celebración prime sobre el dolor. Son necesarios espacios, personas preparadas intelectual y humanamente, disponibilidad real, horarios claros de atención. Pero la atención no sólo debe realizarse 
por una persona sino que debe haber algún momento en que el tema de la diversidad se aborde a nivel de centro (jornadas de formación, de reflexión, de testimonios, de trabajo entre el personal para impulsar iniciativas en el centro). Este tema no es algo privado sino que también tiene una dimensión pública. No tratarlo no sólo limita emocionalmente a los que no son heterosexuales sino que nos limita a todos.

Positiva frente a rechazo. La diversidad sexual hay que vincularla con el bienestar y no con el temor. Todos tenemos que aprender a vivir de forma plural el placer, la comunicación, el afecto, la fecundidad, el deseo, la atracción, el enamoramiento, el encuentro. Ya decía Ellis: "no pienses que la sexualidad de los demás tiene que ser como la tuya". No sólo se trata de respetar diversas ideas sino modos de sentir y ser. Esto supone partir claramente de que la homosexualidad y la disforia de género no son una enfermedad, ni una perversión. Hay que evitar la patologización de la diversidad sexual desde una rígida normalización social. Subrayar en extremo el sufrimiento de su condición refuerza esa normalización social. La diversidad no es una simple elección de una orientación sexual. Es la condición de muchas personas Ilamadas a la plenitud humana desde lo que son. Y, por supuesto, no aparece por hablar de ella, no se contagia, no se favorece por tratarla en las aulas. Nadie cambia su orientación sexual sólo por una influencia externa.

Práctica más que teórica. Los casos concretos de violencia que aparecen en los medios, las narraciones, películas, documentales, materiales lúdicos, páginas web, dinámicas de grupo o representaciones son buenos medios educativos. Hoy no hay auténtica educación si sólo se expone teóricamente, de forma abstracta y alejada de la realidad. No hay que quedarse satisfechos con meras explicaciones teóricas. Hay que cultivar las habilidades interpersonales para modificar la agresividad, la timidez o los bloqueos. Hay que afrontar prácticamente la negación, denigración y discriminación de cualquier forma no heterosexual de comportamiento, identidad, relación. Sigue habiendo vio-
No se puede pactar con el silencio. Gais, lesbianas y transexuales no son aquellos que pueden ser injuriados en cualquier momento. Por eso también es importante hacer públicas medidas disciplinarias concretas ante el insulto, la discriminación 0 la violencia, medidas de defensa de los que son diferentes, no sólo de cara al centro sino ante las familias de los alumnos y la sociedad

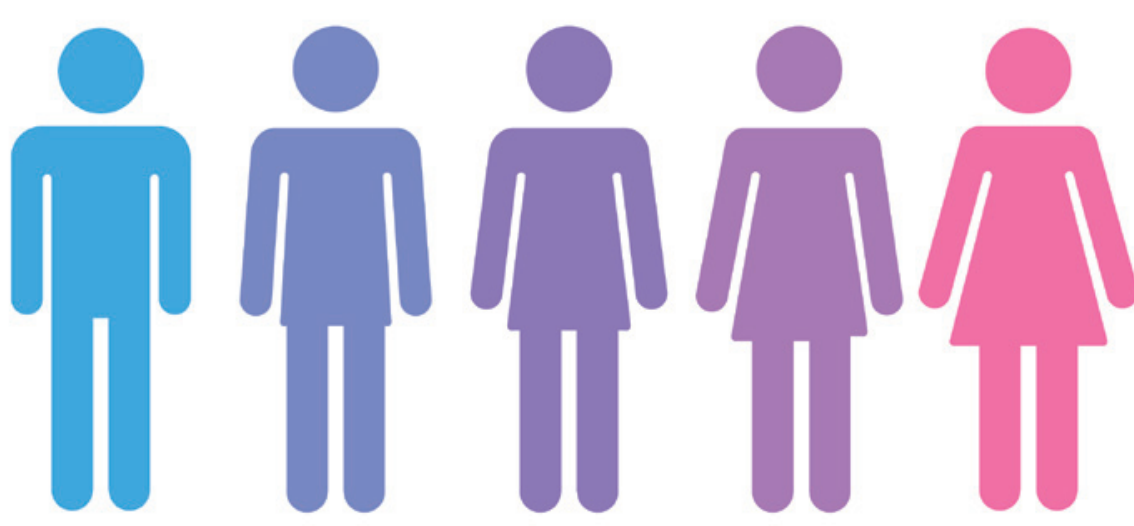

lencia hacia más de la mitad de los gais, lesbianas y transexuales españoles. Los alumnos siguen hoy recibiendo actitudes negativas e información distorsionada desde las familias, los propios compañeros de clase, los amigos o ciertos medios de comunicación. Hay que cambiar la práctica y no sólo los discursos.

Procesual más que estática. La homosexualidad o la disforia de género emergen en la conciencia del sujeto en distintas etapas de la vida y es necesario respetar los diferentes caminos de conformación de la propia identidad sexual. Algunos lo descubren en la infancia, otros en la adolescencia y unos cuantos en la adultez. En ese camino algunos momentos son de incertidumbre y duda, de tanteos y angustias, de vergüenza o de estigma con muy diversas emociones que son resueltas de modo diverso antes de aceptar esa identidad sexual como un dato de sí mismos. La construcción de la identidad homosexual pasa por diversos procesos como la autodefinición, la revelación a otros, la socialización y la aceptación que pueden durar más o menos en el tiempo. En los estudios de seguimiento 
Criterios Educativos

\section{MÍNIMOS DE JUSTICIA EXIGIBLES A TODOS}

\section{INTEGRAR UNOS ROLES DE GÉNERO MÁS FLEXIBLES Y UNA MAYOR} DIVERSIDAD SEXUAL

3. AGOMPAÑAR LA GONSTRUGCIÓN DE LAS DIVERSAS IDENTIDADES SEXUALES

\section{AGOMPAÑAR LA GONGIENCIAY SUS DECISIONES}

de niños prepúberes que fueron remitidos a las clínicas para evaluación de disforia de género, la disforia persistió hasta la edad adulta en sólo 6-23 \% de los niños aunque en algunos niños estos sentimientos se intensifican. La búsqueda de la identidad nunca es un camino lineal, no hay procesos automáticos sino personas. La mayoría de los adolescentes son personas que están en búsqueda, "¿lo soy o no lo soy?", y que comienzan a tener sus propias vivencias sexuales que pueden o no ayudar a la búsqueda, "me siento homosexual pero mi primera experiencia fue un fracaso".

\section{Criterios educativos \\ 1. Mínimos de justicia exigibles a todos}

El artículo 14 de nuestra Constitución reconoce el derecho a no ser discriminado por ninguna "circunstancia personal y social". Hay que educar en la igualdad en la paridad del género masculino y femenino, desterrar el sexismo desde las aulas, evitar la invisibilidad de las contribuciones de las mujeres (ciencia, empresa), educar en el valor de reivindicar la igualdad. Esto supone poner el foco en la discriminación y la emancipación de la mujer y también poner, en algunas ocasiones, límites al excesivo poder masculino tanto a nivel de pareja, instituciones, sociedad. La cultura, la sociedad, las prácticas sociales y el simbolismo cul- tural influyen en la constitución de la identidad y de las relaciones sexuales. No hay un mero determinismo biológico. Por este motivo siempre hay que estar alerta para que la cultura y la sociedad no impongan determinadas identidades en virtud de determinadas relaciones de poder y roles sociales marginando y discriminando a mujeres, a homosexuales, a transexuales, etc. La cultura puede ser muy dura y cruel con los que no se reconocen como heterosexuales. ¿Qué implica esto?

Hay que conocer y valorar críticamente las injusticias en el trato de las personas, la violencia, la discriminación. No se puede dejar de enseñar la injusticia que supone que actualmente seis países prevén la pena de muerte como castigo para las relaciones homosexuales y que sólo a partir de 1960 se empiezan a tolerar las relaciones homosexuales en privado en algunos pocos países.

Atender la diversidad supone que hay que denunciar el acoso escolar y la violencia a personas homosexuales y transexuales (y también a personas de otras etnias, clase social, religión, etc.). Muchos tienen miedo de denunciar. En Estados Unidos (2011), el 78 \% de las personas no "convencionales" en cuanto a su género sufre acoso escolar. El sufrimiento puede ser tal que lleva a "demasiadas" personas al suicidio.

Atender la diversidad implica que en nuestros centros no se pueden tolerar un lenguaje discriminatorio ni chistes sobre maricas, maricones o bolleras (ni gitanos, ni musulmanes, ni latinos, etc.). Hay que ser muy cuidadosos con el lenguaje que empleamos. No caben caricaturas. No hay que dejar pasar la oportunidad pedagógica ante una burla. No se puede pactar con el silencio. Gais, lesbianas y transexuales no son aquellos que pueden ser injuriados en cualquier momento. Por eso también es importante hacer públicas medidas disciplinarias concretas ante el insulto, la discriminación o la violencia, medidas de defensa de los que son diferentes, no sólo de cara al centro sino ante las familias de los alumnos y la sociedad. 


\section{La atención de la diversidad supone finalmente atender a sus relaciones y parejas, reconocer uniones de amor más allá de lo normalizado, reconocer que hay parejas excepcionales}

\section{Integrar roles de género más flexibles y una mayor diversidad sexual}

Más allá de la justicia, la escuela debe abrir a todos la realidad de la diversidad sexual. No siempre la dimensión biológica, psicológica y social de la sexualidad se integran armónicamente. Esto es claro en los procesos de socialización de personas con malformaciones en los órganos genitales en el nacimiento o en su deterioro en los primeros años de vida, en individuos intersexuales o en algunos niños y jóvenes cuya sensibilidad y gustos no se ajustan a los de la mayoría. La antropología cultural nos enseña el modo diverso que tienen las sociedades de interpretar los cuerpos sexuados y los roles. Las diferencias varón-mujer no corresponden a una naturaleza cerrada y dada.

Hay que asumir una pluralidad de géneros más allá del masculino heterosexual y el femenino heterosexual. Hay que reconocer y valorar las distintas y diversas masculinidades y las distintas y diversas feminidades. Hay que apoyar públicamente los procesos de los jóvenes con una identidad u orientación sexual no normativa, apoyar su modo de ser, sus capacidades y gustos, hacerlos sentir orgullosos de sí mismos y fuertes frente a la mirada social, armarles contra los más que posibles rechazos. Y también invitarlos a considerar que si adoptan un comportamiento más adaptado a las presiones sociales están internalizando una valoración negativa, están dejando a un lado posibles dones que tienen y están claudicando ante los dictados de las mayorías.

\section{ACTIVIDADES DE AULA}

\section{Actividad sobre narraciones de roles de género e insultos ( $1^{0} \mathrm{de}$ ESO)}

\section{Completa el final de la historia. ¿Y tú cómo seguirías?}

Las narraciones y cuentos son de los recursos mejores en últimos años de Primaria y primeros de Secundaria para empezar a educar en la diversidad en torno a insultos, roles de género y diversidad sexual. Lo importante es ir cambiando las historias según la edad, los contextos y las problemáticas. Algunas de las más básicas para partir serían:

ע María tiene 12 años y no le gustan los vestidos. Siempre va en vaqueros. Una amiga la ha invitado a un cumpleaños el sábado y le ha dicho que vaya formal. El viernes por la noche, la madre intenta convencerla para que lleve vestido en vez de pantalones...

У Juan está en primero de Primaria y disfruta jugando con muñecas y diademas en el recreo con sus compañeros y compañeras de clase. Su hermano Víctor, de sexto de Primaria lo ve jugando, le grita y le dice que parece una "niñaza". Un amigo de Víctor interviene...

$\checkmark \quad$ Luis tiene 14 años y nunca ha salido con chicas. No sabe lo que le pasa. № sabe si la atracción que siente por Francisco es algo especial o no. Se siente a gusto con él. No se sabe si es algo pasajero. Sus otros amigos empiezan a hacer bromas de él y cómo se acerca a Francisco...

Hay que atender muy especialmente a los alumnos inmigrantes. Su sexualidad es vivida de modo muy diverso a la nuestra. Hay que aprovechar lo bueno de las diversas culturas ofreciendo también una reflexión sobre los derechos humanos y su universalidad.

\section{Acompañar la construcción de las diversas identidades sexuales}

Los individuos pueden sentir la necesidad de criticar, reformular, reconfigurar y hasta alterar la identidad o el rol recibidos socialmente. Hay dimensiones de la identidad que tienen que ver con el cuidado de los niños, el engendrar niños, amamantar, dar y recibir placer sexual, la sanción de las emociones, la división del trabajo, la gestión del poder, etc. que pueden y, en muchas ocasiones, deben reformularse. En estos procesos de construcción y reformulación de la identidad es importante educar al alumnado homosexual y transexual con referentes y modelos positivos. Esto implica subrayar que ciertos gais, lesbianas y transexuales han hecho aportaciones muy positivas a la sociedad, que muchos viven su sexualidad de forma adulta y madura y que es importante que cuiden de su propia autoestima, 
su propia dignidad, su propio cuerpo, su propio sentir por encima de ser sexualmente de un tipo o de otro (son personas valiosas por encima de lo sexual).

Los educadores deben hacer un esfuerzo por comprender lo que el alumno vive. Sus sentimientos deben ser respetados y no se deben despreciar diciendo que se pasan y se olvidan con el tiempo. En algunos casos será así, pero en otros casos no. Se les debe transmitir confianza, que su orientación e identidad es válida, natural, respetable y que con ella pueden alcanzar la felicidad. Hay que ayudarles a estimar sus deseos y sentimientos, sus preferencias y gustos. Lo importante es que los sentimientos de duda, angustia y soledad que experimentan en este proceso de búsqueda desparezcan lo más pronto posible. Por eso es fundamental acompañarlos en este proceso. En este acompañamiento es bueno integrarlos en proyectos más amplios donde descubran su valor como matemáticos, literatos, educadores, etc.

\section{Acompañar la conciencia y sus decisiones}

Es importante acompañar los procesos de búsqueda individual mostrándoles reconocimiento y afecto, confianza y escucha. Estos procesos pueden afectar a su rendimiento escolar. El acompañamiento es esencial en un ámbito donde hay temor ante la respuesta de la familia, la pérdida de apoyos económicos, el rechazo de sus amigos, la marginación en el colegio, la sensación de que no tendrán familia en el futuro, etc. Los alumnos ne-

\section{DC) DARA SABER MÂS}

Pichardo Galán, J. I. (2009). Adolescentes ante la diversidad sexual: homofobia en los centros educativos. Madrid: La Catarata.

SÁnchez, M. (2009). Cómo educar en la diversidad afectivo-sexual en los centros escolares. Madrid: La Catarata. cesitan personas con las que puedan hablar sobre sus miedos y su situación.

Los adolescentes gais, lesbianas y transexuales necesitan ayuda en el momento de reconocerse como tales ante los demás. No es fácil tener que ocultar los sentimientos. Pueden ser momentos complicados en los que necesitan personas de confianza para que en las dificultades su desarrollo y autoestima no se quiebren, para compartir experiencias. Son decisiones personales (salir del armario) que deben tomar ellos y que de ningún modo se pueden violentar. No se puede obligar a decirlo, ni hay que decirlo a todo el mundo.

El apoyo a las familias es importante en el momento de descubrimiento de la homosexualidad o transexualidad del hijo. Estos momentos pueden originar fuertes crisis familiares y deben ser apoyadas. La mayoría pasan por diversos momentos como la negación (ya se te pasará), la ira (ante los nuevos amigos), la conmoción, la negociación (no me importa, pero no vayas a determinados sitios), la angustia (el estigma de ser padre de un homosexual), la tristeza (por las expectativas frustradas), la culpa (no lo hemos detectado a tiempo) y la aceptación.

La atención de la diversidad supone finalmente atender a sus relaciones y parejas, reconocer uniones de amor más allá de lo normalizado, reconocer que hay parejas excepcionales. Hay que cultivar una mirada que se alegra porque se amen. Su amor ni es pecaminoso, ni fingido, ni despreciable. Es real. Es un regalo para todos

HEMOS HABLADO DE

\section{Diversidad sexual; identidad sexual; acompañamiento; educación sexual.}

Este artículo fue solicitado por PADRES Y MAESTROS en mayo de 2017, revisado y aceptado en octubre de 2017. 